\title{
Improved Background Correction for the Quantification of Actinide M-lines in EPMA
}

\author{
Xenia Ritter ${ }^{1,2}$, Philipp Pöml ${ }^{1}$, Stéphane Brémier ${ }^{1}$, Jasper Berndt ${ }^{2}$ \\ 1. European Commission, Joint Research Centre, Institute for Transuranium Elements, P.O. Box \\ 2340, 76125 Karlsruhe, Germany \\ 2. Institut für Mineralogie, Westf. Wilhelms-Universität, Corrensstr. 24, 48149 Münster, Germany
}

Electron probe micro-analysis (EPMA) is an important technique for a broad range of applications in nuclear sciences. One main target is to improve the safety of the nuclear fuel cycle, by studying the chemical and physical properties of spent nuclear fuel and its fission products, either solids, volatiles, or gases, after the irradiation [1]. Of particular interest for the nuclear scientist is the distribution and quantity of actinides in the fuel, either before or after irradiation. Actinides in nuclear fuel can be added during fuel fabrication (usually containing the fissile material), or are being produced during irradiation by neutron capture and/or alpha-decay of existing actinides. The fuel types most commonly studied by EPMA are uranium oxide and Mixed Oxide Fuel (MOX) containing a mixture of uranium and plutonium oxide.

There are other concepts of nuclear fuel, e.g., thorium based compounds, or special target fuels for the burning of unwanted minor actinides $(\mathrm{Np}, \mathrm{Am}, \mathrm{Cm})$, a process known as transmutation. Minor actinides provide a major contribution to the radiotoxicity of spent nuclear fuel. To reduce the spent nuclear fuel's radioactive inventory, the fuel can be reprocessed and minor actinides and fission products separated. The minor actinides can then be mixed with the source materials for fresh nuclear fuel and subsequently be irradiated in a fast neutron reactor. The fast neutrons will transmute part of the minor actinides into nuclides with a short half-life that will then decay to less dangerous or even stable nuclides. For the latter case, EPMA is of particular interest as an analytical tool, because it provides information about the behaviour and level of transmutation of the added minor actinides.

In EPMA, the M-lines $(\sim 4.1-3.3 \AA)$ are used for the quantification of the actinides. They are commonly measured using PET crystals (pentaerythritol, reflecting plane 002, $2 \mathrm{~d}=8.742 \AA$ ). In nuclear sciences, however, spectrometers are often equipped with $\alpha$-quartz crystals (reflecting plane $10 \overline{1} 1,2 \mathrm{~d}=6.686 \AA$ ). Quartz crystals have the advantage of a better energy resolution of the actinide peaks at the cost of intensity. Considering the complexity of the actinide M-line region, however, the gain in resolution is much preferred over the loss in intensity.

The analysis of actinides is complex. Their electron shells produce a variety of X-ray M-lines, all in the same wavelength region. Consequently the lines are overlapping and the analysis of a material containing 2 or more actinides becomes troublesome. Additionally, also the background correction becomes very difficult. Fig. 1 shows as an example a $\mathrm{PuO}_{2}$ sample containing a small amount of Am. The number and density of M-lines results in an increased background, that is not following the continuum trend anymore. It is hence not possible to use the general background correction approach of just measuring the background left and right of the peak and linearly interpolating the intensity. On top of that, the Ar K absorption edge (3.871 $\AA$ ) falls exactly into this region, causing a change of both peak and background intensities (see Fig. 1). X-ray counters on wavelength dispersive (WD) spectrometers are generally gas flow proportional counters that are filled with $90 \%$ argon - $10 \%$ methane (P10) gas. 
For accurate analysis the background correction problem has to be solved. One approach would be to use the Mean Atomic Number (MAN) approach as described in [2]. However, to calibrate the continuum it is necessary to find standards not containing the element of interest that have both higher and lower mean atomic numbers compared to the unknown. For materials like $\mathrm{UO}_{2}$ or $\mathrm{PuO}_{2}$ this seems impossible to accomplish, because materials like $\mathrm{BkO}_{2}$ are not available.

We hence decided to follow another approach, the multi-point background acquisition developed by Probe Software, Inc. [3] and described in more detail in [4]. This approach allows the analyst to measure multiple background points in the region of interest followed by automatic evaluation and regression. An example is shown in Fig. 1. In this case, a WD spectrum was acquired for the Pu and Am M-line range. From the spectrum, two background points were selected on the far left and two on the far right of the M-peak range, avoiding the increased intensity region and the sharp drop of intensity due to the Ar K absorption edge in the central part. Fitting these points (in this case exponentially, red curve in Fig. 1) much improved background values could be interpolated. The benefit of this approach is that it costs only a little extra time, while improving background correction significantly.

In our contribution we will show WD scans for the actinides Th, $\mathrm{U}, \mathrm{Np}, \mathrm{Pu}$, and $\mathrm{Am}$. We will show spectra of samples containing these as major elements and discuss how to improve background correction considering a high gain in accuracy and little loss of time. In addition, our approach will be tested on a real irradiated fuel sample and the results discussed.

\section{References:}

[1] C Walker, Journal of Analytical Atomic Spectrometry 14 (1999) p.447.

[2] JJ Donovan and TN Tingle, Journal of Microscopy and Microanalysis 2 (1996) p.1.

[3] Probe Software, Inc., 885 Crest Dr., US-97405 Eugene, Oregon, U.S.A.

[4] JM Jercinovic et al, IOP Conf. Series: Materials Science and Engineering 32 (2012) p.012012.

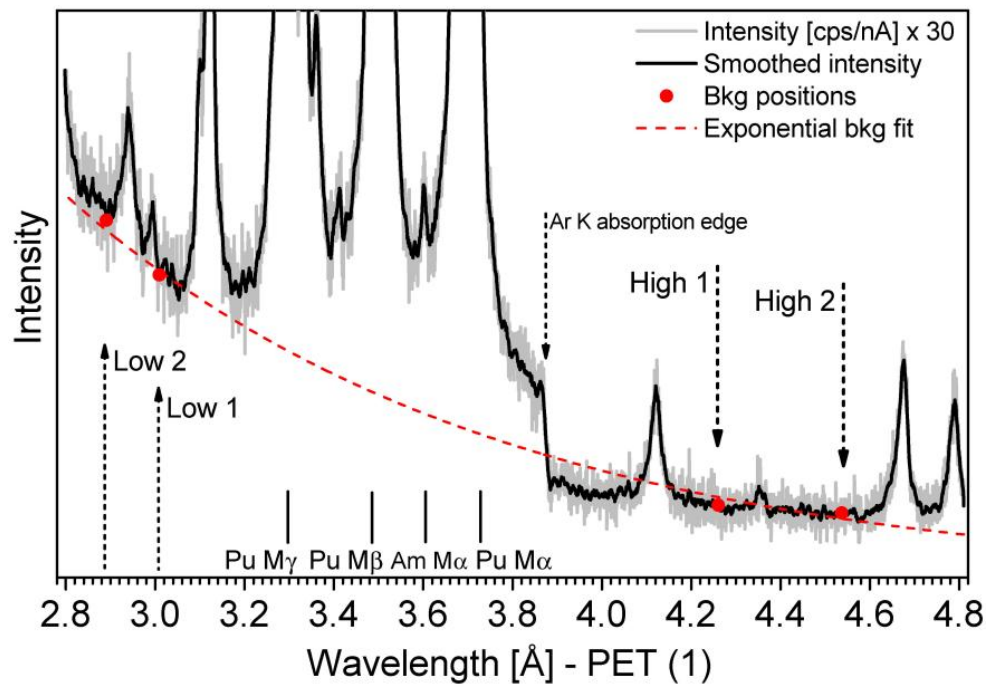

Figure 1. M-line region of a $\mathrm{PuO} 2$ sample containing minor amounts of Am. Red points mark the four selected background positions, the red dashed curve is the regression curve. Note the significant drop in intensity in the centre of the spectrum, due to the Ar K absorption edge. 